\title{
One ion to catch them all: Targeted high-precision Boltzmann thermometry over a wide temperature range with $\mathrm{Gd}^{3+}$
}

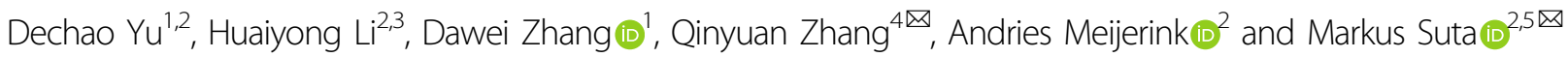

\begin{abstract}
Ratiometric luminescence thermometry with trivalent lanthanide ions and their $4 f^{n}$ energy levels is an emerging technique for non-invasive remote temperature sensing with high spatial and temporal resolution. Conventional ratiometric luminescence thermometry often relies on thermal coupling between two closely lying energy levels governed by Boltzmann's law. Despite its simplicity, Boltzmann thermometry with two excited levels allows precise temperature sensing, but only within a limited temperature range. While low temperatures slow down the nonradiative transitions required to generate a measurable population in the higher excitation level, temperatures that are too high favour equalized populations of the two excited levels, at the expense of low relative thermal sensitivity. In this work, we extend the concept of Boltzmann thermometry to more than two excited levels and provide quantitative guidelines that link the choice of energy gaps between multiple excited states to the performance in different temperature windows. By this approach, it is possible to retain the high relative sensitivity and precision of the temperature measurement over a wide temperature range within the same system. We demonstrate this concept using $\mathrm{YAl}_{3}\left(\mathrm{BO}_{3}\right)_{4}(\mathrm{YAB}): \mathrm{Pr}^{3+}{ } \mathrm{Gd}^{3+}$ with an excited ${ }^{6} \mathrm{P}$, crystal field and spin-orbit split levels of $\mathrm{Gd}^{3+}$ in the UV range to avoid a thermal black body background even at the highest temperatures. This phosphor is easily excitable with inexpensive and powerful blue LEDs at $450 \mathrm{~nm}$. Zero-background luminescence thermometry is realized by using blue-to-UV energy transfer upconversion with the $\mathrm{Pr}^{3+}-\mathrm{Gd}^{3+}$ couple upon excitation in the visible range. This method allows us to cover a temperature window between 30 and $800 \mathrm{~K}$.
\end{abstract}

\section{Introduction}

Chemical reactions, biological functionalities, and various physical phenomena are all strongly determined by the local temperature. The measurement of local temperatures on successively smaller scales requires new methods of temperature sensing that rely on a remote detection principle. Several such concepts already exist.

\footnotetext{
Correspondence: Qinyuan Zhang (qyzhang@scut.edu.cn) or Markus Suta (markus.suta@hhu.de)

'Engineering Research Center of Optical Instrument and System, The Ministry of Education, Shanghai Key Laboratory of Modern Optical Systems, University of Shanghai for Science and Technology, Shanghai 200093, China ${ }^{2}$ Condensed Matter and Interfaces, Debye Institute for Nanomaterials Science, Department of Chemistry, Utrecht University, Princetonplein 1, 3584 CC Utrecht, The Netherlands

Full list of author information is available at the end of the article
}

The most commonly known example is infrared (IR) thermography or pyrometry ${ }^{1-3}$, which only allows temperature measurements of surfaces of objects based on their emitted thermal radiation. According to the Stefan - Boltzmann law, the intensity of the emitted radiation scales with the fourth power of temperature and is expected to be sensitive for high temperatures and objects with high emissivity.

An alternative method of remote temperature sensing is luminescence thermometry, which exploits the fact that luminescence properties such as the emission intensity or the luminescence decay time are highly dependent on the local temperature of the surroundings of the luminescent species $^{4-6}$. It requires a simple setup consisting of a laser excitation source, luminescent micro- or nanocrystals or

\section{() The Author(s) 2021}

(c) (i) Open Access This article is licensed under a Creative Commons Attribution 4.0 International License, which permits use, sharing, adaptation, distribution and reproduction cc) in any medium or format, as long as you give appropriate credit to the original author(s) and the source, provide a link to the Creative Commons license, and indicate if changes were made. The images or other third party material in this article are included in the article's Creative Commons license, unless indicated otherwise in a credit line to the material. If material is not included in the article's Creative Commons license and your intended use is not permitted by statutory regulation or exceeds the permitted use, you will need to obtain permission directly from the copyright holder. To view a copy of this license, visit http://creativecommons.org/licenses/by/4.0/. 
molecules embedded in the object of interest, and a fast and efficient light detection system $^{7-9}$. Its promising advantages over thermography include the lower accessible temperature ranges and non-invasive temperature detection below surfaces. Its successful application in biological media has been highlighted for many different optical centres, such as nitrogen vacancy $\left(\mathrm{NV}^{-}\right)$centres in fluorescent nanodiamonds ${ }^{10-12}$, semiconducting quantum dots $(\mathrm{QDs})^{13-15}$, bulk halides ${ }^{16,17}$, and organic fluorophores $^{18-21}$.

An alternative emerging class of luminescent thermometers are micro- or nanocrystals doped with trivalent lanthanide ions ${ }^{22,23}$. Their rich electronic level structure that arises from the partly filled $4 f^{n}(n=1-14)$ inner shell with characteristic energy gaps on the order of thermal energies (several $100 \mathrm{~cm}^{-1}$ ) allows for thermal coupling between energetically adjacent levels. Most representatives of this class of luminescent thermometers thus rely on ratiometric sensing; in ratiometric sensing, the temperature is measured by means of the intensity ratio of the emission bands from two thermally coupled excited levels. The key advantages of this approach are its simplicity and that it is self-referenced. Moreover, the narrow linewidths of the intraconfigurational $4 \mathrm{f}^{n}-4 \mathrm{f}^{n}$ transitions minimize spectral overlaps between emission lines from two thermally coupled energy levels, even when the energy separation is small. The approximate independence of their energies from the surrounding host material related to the low radial extension of the $4 \mathrm{f}$ orbitals allows us to select the emission energy range of interest by appropriately choosing the lanthanide ion. Recently, there has also been progress in reverting this scheme and performing thermometry by detecting a reference emission from the same excited level upon dual excitation from two thermally coupled ground levels ${ }^{24-26}$. Luminescence (nano)thermometry has been demonstrated to be both a precise and accurate technique for probing fundamental thermodynamic phenomena at the micro- and nanoscale $^{27-29}$ and helps to assess the local temperature fluctuations in tissue $\mathrm{e}^{30-35}$ or in chemical reactors ${ }^{36-40}$. Currently, there has been observable progress on how to implement and standardize this technique for applications $^{41-45}$, the connected limitations ${ }^{46,47}$, or how to couple temperature sensing with other functionalities such as single-molecule magnetism ${ }^{48,49}$ and (meso-)porous materials $^{50,51}$, e.g., theranostics ${ }^{52}$.

The intensity ratio of the emission lines originating from two thermally coupled excited levels within the same configuration follows simple Boltzmann statistics if thermalization is faster than population decay (by any radiative or nonradiative pathways) ${ }^{53,54}$. It has been recently demonstrated that despite their appealing simplicity, Boltzmann-based thermometers with two excited levels suffer from a fundamental thermodynamic limitation ${ }^{55}$.
At very low temperatures compared to the energy gap $\Delta E_{21}$ between the two excited levels $|1\rangle$ and $|2\rangle\left(k_{\mathrm{B}} T \ll\right.$ $\left.\Delta E_{21}\right)$, the probability of thermally exciting the population from the lower to the higher excited emissive level is vanishingly small, which translates to a negligible intensity of the emission from the higher excited level. In addition, the nonradiative absorption rate governing the thermalization from level $|1\rangle$ to $|2\rangle$ becomes so slow that it can no longer compete with the radiative decay of level $|1\rangle$, which leads to a decoupling of the two excited states. In contrast, at very high temperatures compared to the energy gap $\left(k_{\mathrm{B}} T \gg \Delta E_{21}\right)$, the populations of the two excited levels in the thermodynamic limit effectively equalize and thus lower the sensitivity of the thermometer, which relies on a relative net change in population between the two excited levels. Both described extremes diminish the overall achievable precision of the thermometer and imply that any Boltzmann-based two-level thermometer only performs with sufficient statistical precision within a limited temperature range dependent on the energy gap $\Delta E_{21}$ between the two excited levels.

A way to circumvent this thermodynamic limitation and to widen the temperature range for the highest precision of a Boltzmann-based thermometer is to extend the concept of thermal coupling between more than two energetically close excited levels. This method allows us to retain both an optimized response and sensitivity of a single luminescent thermometer by monitoring emission intensity ratios from multiple higher energy excited levels separated by different energy gaps from the lowest excited emissive state. While this has already been realized on a qualitative level ${ }^{56-58}$, no clear guidelines have been established with regard to the most advisable temperature ranges for energy gaps for two or more higher excited levels thus far. Knowledge of the fundamental thermodynamic properties of such a thermally coupled multilevel Boltzmann-based luminescent thermometer helps decide at which temperatures a change to another luminescence intensity ratio (LIR) is advisable to achieve the highest thermometry precision and which combination of energy gaps is optimal for the (wide) temperature range of interest.

Luminescence thermometry at high temperatures also suffers from another practical problem. Any solid emits thermal or black-body radiation, which becomes more intense at high temperatures. Moreover, Wien's displacement law $\left(\lambda_{\max } T=b\right)$ with $\left.b \approx 2.8978 \mathrm{~mm} \cdot \mathrm{K}\right)$ states that the peak wavelength of the Planckian black-body spectrum shifts to shorter wavelengths at high temperatures. While the peak maximum clearly lies in the infrared range at usual lab-accessible temperatures $(T<2000 \mathrm{~K})$, the short-wavelength tail of the black-body spectrum can already interfere with the luminescence spectrum in the visible range at temperatures as low as $800 \mathrm{~K}$. Thus, any 
ratiometric luminescent thermometer devised for high temperature sensing best relies on emission in the ultraviolet range, which is clearly unaffected by black-body background at temperatures below $1200 \mathrm{~K}$.

In this work, we present such a designed multilevel luminescent thermometer that uses the three excited ${ }^{6} \mathrm{P}_{J}$ $(J=7 / 2,5 / 2,3 / 2)$ crystal fields and spin-orbit levels of the UV B-emitting lanthanide ion $\mathrm{Gd}^{3+}$; we were motivated by thermodynamic considerations and demonstrated how a single luminescent phosphor can be optimized for precise thermometry from cryogenic $(30 \mathrm{~K})$ to high temperatures $(800 \mathrm{~K})$ with constantly high relative sensitivities above $0.5 \% \mathrm{~K}^{-1}$. Intense excitation with a costeffective blue wavelength of $450 \mathrm{~nm}$ is interesting for practical applications. This is possible by co-doping with $\mathrm{Pr}^{3+}$ in huntite-type crystallizing $\mathrm{YAl}_{3}\left(\mathrm{BO}_{3}\right)_{4}(\mathrm{YAB})^{59}$ and upconversion into its $4 \mathrm{f}^{1} 5 \mathrm{~d}^{1}$ configuration ${ }^{60-64}$, followed by efficient energy transfer to the $\left.{ }^{6} \mathrm{I}_{\gamma^{\prime}}{ }^{\prime}{ }^{\prime}=17 / 2 \ldots 7 / 2\right)$ levels of $\mathrm{Gd}^{3+}$. Not only does this offer the possibility of background free upconversion thermometry, but it also bears potential for applications such as UV lasing ${ }^{64}$ or visible light excited photocatalysis ${ }^{65}$ combined with local temperature sensing. Similar visible-to-UV upconversion relying on triplet-triplet annihilation has otherwise been recently reported by Harada et al. for metal-organic compounds $^{66}$

\section{Results}

\section{Thermometric performance of an excited three-level system}

The benefit of a third emissive level on the performance of a luminescent thermometer can be appreciated as follows. The thermal response (often also referred to as absolute sensitivity), $S_{\mathrm{a}}(T)$, of the luminescence intensity ratio (LIR) is directly related to the absolute effective thermal population of the microstate of the higher excited level from which the emission arises. The relative sensitivity, $S_{\mathrm{r}}(T)$, in contrast, relates to the relative net change in population between the two thermally coupled excited levels of interest ${ }^{55}$. As indicated above, the thermodynamically optimized performance of a two-excited level thermometer is limited to a small temperature range only. This performance is related to the low response at low temperatures due to the almost negligible thermal population in the higher excited state and a low relative sensitivity at high temperatures due to almost equalized populations (per microstate) in both thermally coupled excited levels. The optimum temperature range depends on the probed energy gap $\Delta E_{21}$ and is given by ${ }^{55}$

$$
T_{\mathrm{opt}} \in\left[\frac{\Delta E_{21}}{(2+\sqrt{2}) k_{\mathrm{B}}}, \frac{\Delta E_{21}}{2 k_{\mathrm{B}}}\right]
$$

A simple way to widen this optimum temperature range is the addition of a third excited level $|3\rangle$ separated by an energy gap $\Delta E_{31}=(1+s) \Delta E_{21}$ from the lowest excited level $|1\rangle$. According to Eq. (1), a widening of the optimum temperature range can be achieved if $s \geq \sqrt{2} / 2 \approx 0.707$ and $\Delta E_{31}$ is used as the thermometric measure above the critical temperature $T^{\prime}=\frac{\Delta E_{21}}{2 k_{k}}$.

This proposal is schematically depicted in Fig. 1a-c. Above $T^{\prime}$, the relative net change in population between levels $|3\rangle$ and $|1\rangle$ becomes higher than the respective net change between levels $|2\rangle$ and $|1\rangle$, resulting in a higher relative sensitivity of the thermometer upon the exploitation of the higher energy gap. This strategy can also be generalized to more than three levels in an iterative manner.

\section{Boltzmann cryothermometry with $\mathrm{Gd}^{3+}$}

The previously introduced thermodynamic concept of a multilevel thermometer can be realized with $\mathrm{Gd}^{3+}$. Here, we use the small energy splitting between different crystal field components of the ${ }^{6} \mathrm{P}_{7 / 2}$ level for temperature sensing in the low-temperature regime. In the next section, the larger splitting between ${ }^{6} \mathrm{P}_{7 / 2}$ and higher energetic spinorbit levels ${ }^{6} \mathrm{P}_{5 / 2}$ and ${ }^{6} \mathrm{P}_{3 / 2}$ for accurate measurements at higher temperatures is exploited instead. A schematic energy level diagram illustrating the working principle of crystal field component-based Boltzmann cryothermometry with $\mathrm{Gd}^{3+}$ is depicted in Fig. 2a. For cryogenic temperatures $(4 \mathrm{~K}<T<100 \mathrm{~K})$, Eq. (1) suggests that the most suitable energy gap for the performance of Boltzmann thermometry is on the order of $50-100 \mathrm{~cm}^{-1}$. This energy range is typical for the splitting of $4 \mathrm{f}^{\mathrm{n}}$-based spinorbit levels by the surrounding crystal field potential, although other examples, such as the $R$ lines of $\mathrm{Cr}^{3+}$ doped into hosts with strong crystal fields, also have energy differences on this order of magnitude ${ }^{67-69}$.

$\mathrm{Gd}^{3+}$ is a very weak absorber ${ }^{70,71}$. In addition, efficient and intense UV light sources are not readily available, and direct UV excitation also gives rise to background emission. We thus chose a different strategy by co-doping the sample with $\mathrm{Pr}^{3+}$, which is schematically depicted in Fig. 2b. Upon excitation with a pulsed optical parametric oscillator (OPO) in the blue range at $448 \mathrm{~nm}$, the $\operatorname{Pr}^{3+}$ ions are excited to the ${ }^{3} \mathrm{P}_{2}$ level. After quick temperatureindependent nonradiative relaxation to the ${ }^{1} \mathrm{D}_{2}$ level given the high phonon energies in $\mathrm{YAB}\left(\hbar \omega_{\max } \sim 1300 \mathrm{~cm}^{-1}\right)^{72}$, electric dipole-allowed excited state absorption (ESA) into the $4 \mathrm{f}^{1} 5 \mathrm{~d}^{1}$ configuration of $\operatorname{Pr}^{3+}$ at energies in the range of $40,000 \mathrm{~cm}^{-1}$ can take place ${ }^{72}$, which is followed by an energy transfer to the ${ }^{6} \mathrm{I}_{J}$, levels of $\mathrm{Gd}^{3+}$ at energies of approximately $37000 \mathrm{~cm}^{-1}(270 \mathrm{~nm})$. The dependence of the $\mathrm{Gd}^{3+}$-related emission intensity on the incident power of the OPO also indicates a two-photon upconversion 


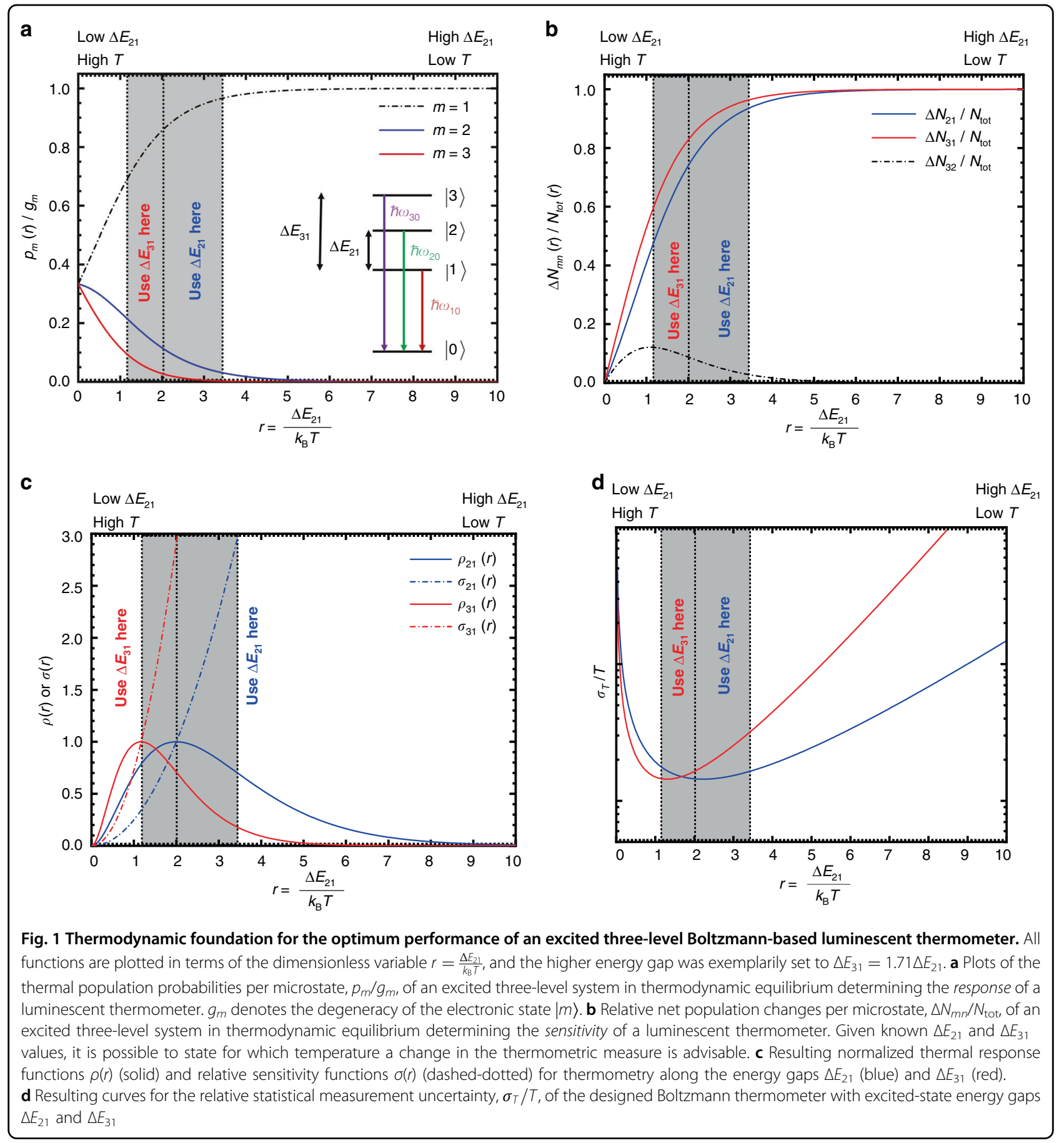

process (see Supplementary Information, Fig. S1). Similar upconversion spectra were, however, also measured using an inexpensive continuous wave $(\mathrm{CW})$ blue laser with $1 \mathrm{~W}$ output power (see Supplementary Information, Fig. S2), which demonstrates that this concept also works with any high-power LED.

Since $\operatorname{Pr}^{3+}$ is very prone to the resonant cross-relaxation process [Pr1, Pr2]: $\left[{ }^{3} \mathrm{P}_{0},{ }^{3} \mathrm{H}_{4}\right] \rightarrow\left[{ }^{1} \mathrm{G}_{4},{ }^{1} \mathrm{G}_{4}\right]$, it was mandatory to keep the $\operatorname{Pr}^{3+}$ concentration at only $0.7 \mathrm{~mol} \%$ (see also Supplementary Information, Fig. S3). The large energy gap between the ${ }^{6} \mathrm{P}_{J}(J=7 / 2 \ldots 3 / 2)$ levels and the ${ }^{8} \mathrm{~S}_{7 / 2}$ ground level of $\mathrm{Gd}^{3+}$, which does not have any intermediate electronic levels, as well as the high mutual lanthanide distances in the YAB host structure $(\sim 5.9 \AA)^{59}$, allow an increase in the $\mathrm{Gd}^{3+}$ concentration to $20 \mathrm{~mol} \%$ without any observable concentration quenching effects (see Supplementary Information, Fig. S4). The high energy gap increases the probability of efficient energy 

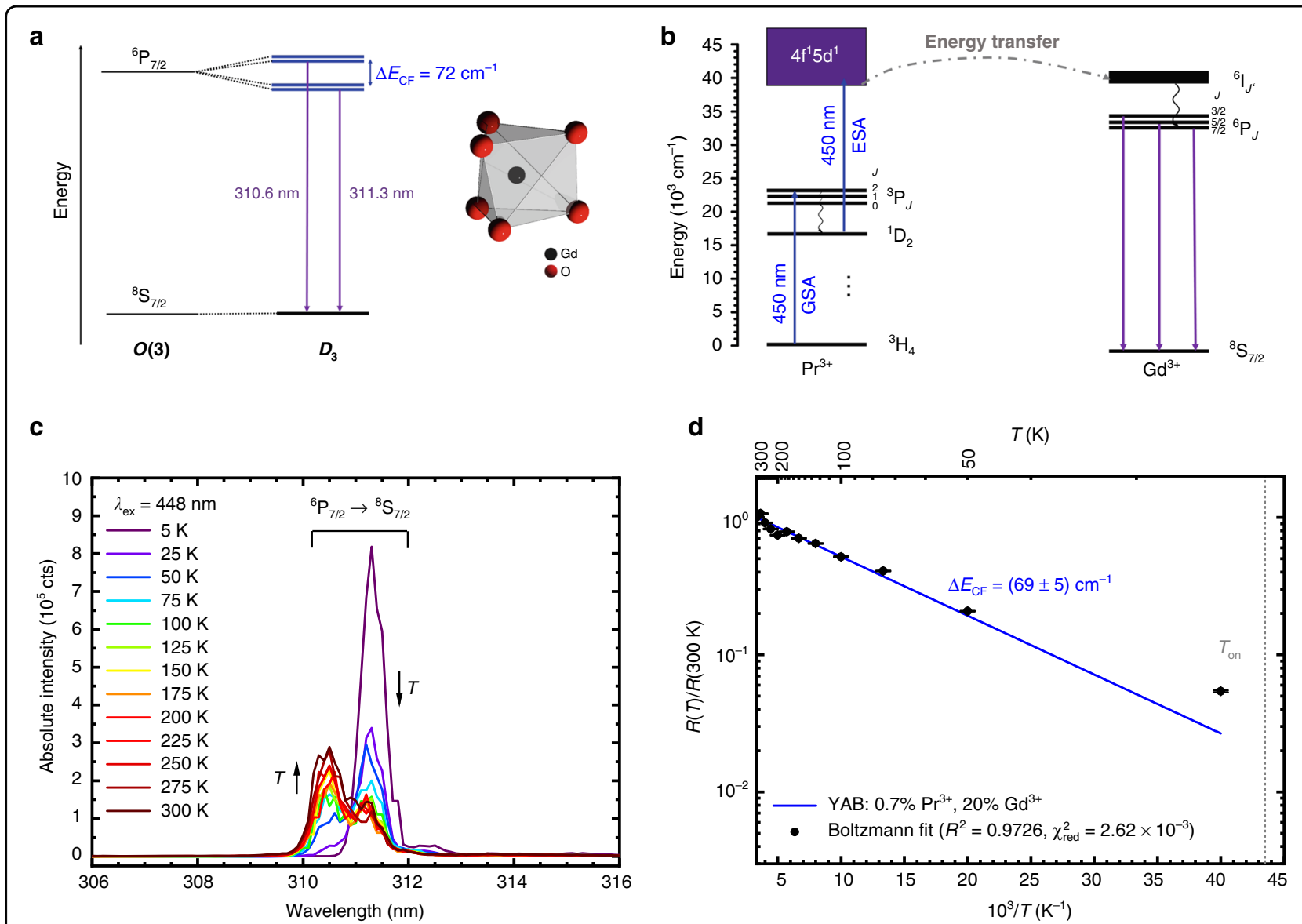

Fig. 2 Thermodynamically optimized Boltzmann cryothermometry with $\mathrm{Gd}^{3+}$ in $\mathrm{YAB}: \mathrm{Pr}^{3+}, \mathrm{Gd}^{3+}$. a Splitting of the lowest excited ${ }^{6} \mathrm{P}_{7 / 2}$ spinorbit level of $\mathrm{Gd}^{3+}$ into the different Kramers' doublets at the present $D_{3}$ site symmetry in YAB. The doped $\mathrm{Gd}^{3+}$ ions are sixfold coordinated in the form of a twisted trigonal prism (inset). $\mathbf{b}$ Indirect upconversion excitation scheme for $\mathrm{Gd}^{3+}$ by energy transfer from the $4 f^{1} 5 d^{1}$-related electronic states of $\mathrm{Pr}^{3+}$. Both the ground state absorption (GSA) and the excited state absorption (ESA) of two $450 \mathrm{~nm}$ photons within the pulse period $(T=50 \mathrm{~ms})$ of the used pulsed laser source are indicated. $\mathbf{c}$ High-resolution upconversion photoluminescence spectra of $\mathrm{YAB}: 0.7 \% \mathrm{Pr}^{3+}, 20 \% \mathrm{Gd}^{3+}$ upon excitation of $\mathrm{Pr}^{3+}$ at $448 \mathrm{~nm}$ showing the temperature dependence of the two radiative transitions from the lower and higher crystal field states of the ${ }^{6} \mathrm{P}_{7 / 2}$ level. The arrows mark the two thermometrically employed transitions. d Boltzmann plot of the temperature-dependent LIR normalized to its value at $300 \mathrm{~K}$. The fitted energy gap, statistical figures of merit, and expected onset temperature for Boltzmann behaviour (see Eq. (5)) are indicated

transfer from $\mathrm{Pr}^{3+}$. Fig. 2c depicts a portion of the luminescence spectra of YAB: $0.7 \% \mathrm{Pr}^{3+}$ and $20 \% \mathrm{Gd}^{3+}$ appear in the UV-B range and clearly show that the strategy to excite $\mathrm{Gd}^{3+}$ via $\mathrm{Pr}^{3+}$-based upconversion and subsequent energy transfer does work. The absence of a $4 \mathrm{f}^{1} 5 \mathrm{~d}^{1} \rightarrow 4 \mathrm{f}^{2}$ broad-band emission of $\mathrm{Pr}^{3+}$ also shows that the energy transfer from $\mathrm{Pr}^{3+}$ to $\mathrm{Gd}^{3+}$ must be very efficient, which is in agreement with earlier findings ${ }^{73}$.

Boltzmann thermometry with $\mathrm{Gd}^{3+}$ at cryogenic temperatures becomes possible if the two clearly resolved components of the ${ }^{6} \mathrm{P}_{7 / 2} \rightarrow{ }^{8} \mathrm{~S}_{7 / 2}$-based group of radiative transitions are chosen (see Fig. 2a). These transitions give rise to two intense emission peaks located at $310.6 \mathrm{~nm}$ and $311.3 \mathrm{~nm}$ (see Fig. 2b), whose intensity ratio (higher energetic to lower energetic emission) is used as the temperature-correlated signal. The LIR at temperature
$T, R(T)$, normalized to its value at the highest measured reference temperature, $T_{0}$, thus follows the law ${ }^{23,74}$

$$
\frac{R(T)}{R\left(T_{0}\right)}=\exp \left[-\frac{\Delta E_{m 1}}{k_{\mathrm{B}}}\left(\frac{1}{T}-\frac{1}{T_{0}}\right)\right]
$$

where $k_{\mathrm{B}}$ is the Boltzmann constant and $\Delta E_{m 1}(m>1)$ is the energy gap between the two thermally coupled levels and is the only fitting parameter. A fit to a linearized version of Eq. (2) gave an effective energy gap of $\Delta E_{\mathrm{CF}}=$ $(69 \pm 5) \mathrm{cm}^{-1}$, in excellent agreement with the value of $72 \mathrm{~cm}^{-1}$ determined from the emission spectra. It should be noted that a corresponding splitting of the ${ }^{8} \mathrm{~S}_{7 / 2}$ level into Kramers' doublets is much smaller (on the order of only $1 \mathrm{~cm}^{-1}$ ) if spin-orbit coupling is only intermediate because of the lack of orbital degeneracy given the 
quantum number $L=0^{75}$. This makes the ${ }^{8} \mathrm{~S}_{7 / 2}$ ground level an effective single level in the case of $\mathrm{Gd}^{3+}$.

The LIR deviates from the expected Boltzmann behaviour at temperatures below $25 \mathrm{~K}$. This observation is related to a kinetic limitation. The nonradiative absorption rate from the lower to the higher excited level, $k_{\mathrm{nr}}^{\mathrm{abs}}(T)$, is given by ${ }^{76,77}$

$$
k_{\mathrm{nr}}^{\mathrm{abs}}(T)=g_{m} k_{\mathrm{nr}}(0)\langle n\rangle^{p}
$$

with $g_{m}$ as the degeneracy of the higher excited level $\left(g_{m}=2\right.$ for Kramers' doublets), $k_{\mathrm{nr}}(0)$ as an intrinsic nonradiative rate governed by the properties of the electronic transition, and

$$
\langle n\rangle=\frac{1}{\exp \left(\frac{\hbar \omega_{\mathrm{ph}}}{k_{\mathrm{B}} T}\right)-1}
$$

as the thermally averaged phonon occupation number of an acoustic or optical phonon mode with energy $\hbar \omega_{\text {ph }}$ and $p$ as the number of phonons consumed during the thermalization between the two excited levels $\left(\Delta E_{m 1}=p \hbar \omega_{\mathrm{ph}}\right.$, here it is $p=1$ ). This temperature-dependent nonradiative absorption rate competes with the radiative decay (and eventually other quenching) rates from level $|1\rangle$. At sufficiently low temperatures, the nonradiative absorption rate decreases than the intrinsic radiative decay rate, and thus, Boltzmann thermalization is kinetically inhibited. We have recently shown that a very accurate estimate for the expected onset temperature of Boltzmann behaviour, $T_{\mathrm{on}}$, is given by ${ }^{55}$

$$
T_{\text {on }}=0.2227 \frac{\Delta E_{m 1}}{k_{\mathrm{B}}}
$$

With the spectroscopic value of $\Delta E_{\mathrm{CF}}=72 \mathrm{~cm}^{-1}$, the expected onset temperature for Boltzmann thermalization between the two excited levels is thus approximately $23 \mathrm{~K}$, very close to the observed deviation from Boltzmann behaviour below $25 \mathrm{~K}$. A similar deviation has also been observed in the case of $\mathrm{Cr}^{3+67,78}$, which can be explained by the same kinetic effect. In turn, the optimum temperature range for the most precise thermometry that exploits this energy gap between the two Kramers' doublets of the ${ }^{6} \mathrm{P}_{7 / 2}$ level of $\mathrm{Gd}^{3+}$ is between 30 and $51 \mathrm{~K}$ according to Eq. (1). The connected relative sensitivities, $S_{\mathrm{r}}(T)$, as defined by

$$
S_{r}(T)=\left|\frac{1}{R(T)} \frac{\mathrm{d} R}{\mathrm{~d} T}\right|=\frac{\Delta E_{m 1}}{k_{\mathrm{B}} T^{2}}
$$

in the case of a Boltzmann thermometer that varies between $S_{\mathrm{r}}(30 \mathrm{~K})=11.6 \% \mathrm{~K}^{-1}$ and $S_{\mathrm{r}}(51 \mathrm{~K})=3.98 \% \mathrm{~K}^{-1}$ in this optimum range, which is clearly above the usually desired threshold of $S_{\mathrm{r}}=1 \% \mathrm{~K}^{-1}$ in practice ${ }^{23}$. In fact, the relative sensitivity at $30 \mathrm{~K}$ is the highest reported value for luminescent cryothermometers at that particular temperature thus far ${ }^{50,67,68,79,80}$. It is important to note, however, that it is both the balance between reliably detectable luminescence signals and relative sensitivity that determine the overall precision of a luminescent thermometer (see also below) ${ }^{55,81}$. Another important point is that for accurate cryothermometry with small energy gaps, a high spectral resolution spectrometer is also required to separate two emission lines that are close in wavelength. Finally, that high relative sensitivities at cryogenic temperatures are not surprising, as can already be appreciated from the $T^{-2}$ dependence in Eq. (6) that dominates at low temperatures and stems from the strong relative net change in population between the two thermally coupled excited levels in this domain (see Fig. 1b).

\section{Extension of high-precision thermometry with $\mathrm{Gd}^{3+}$ to higher temperatures}

The small crystal field splitting between the Kramers' doublets stemming from the ${ }^{6} \mathrm{P}_{7 / 2}$ spin-orbit level of $\mathrm{Gd}^{3+}$ is not well suited for temperature measurements above $100 \mathrm{~K}$ since the excited state populations in the two thermally coupled crystal field states equalize at elevated temperatures. This fact in turn lowers both the response and sensitivity of the chosen radiative transitions for temperature sensing. For thermometry in the range of room temperature, it is necessary to employ two levels with a higher energy gap. According to Eq. (1), a suitable energy gap to measure temperatures between 200 and $400 \mathrm{~K}$ is on the order of $500-700 \mathrm{~cm}^{-1}$, in the range of spin-orbit interaction-induced splitting for the $4 \mathrm{f}^{\mathrm{n}}$-based electronic levels of lanthanides. Consequently, we used the LIR of the radiative transitions from the ${ }^{6} \mathrm{P}_{7 / 2}$ and ${ }^{6} \mathrm{P}_{5 /}$ ${ }_{2}$ spin-orbit levels to the ${ }^{8} \mathrm{~S}_{7 / 2}$ ground level (see Fig. 3a) of $\mathrm{Gd}^{3+}$ to demonstrate its applicability for temperature sensing in the range of room temperature (see Fig. 3b). The LIR of the ${ }^{6} \mathrm{P}_{5 / 2}$ to ${ }^{6} \mathrm{P}_{7 / 2}$ emission lines was measured as a function of temperature as determined from the emission spectra shown in Fig. $3 \mathrm{~b}$ and fit to Eq. (2) (Fig. 3c). The results indicated an effective energy gap between the ${ }^{6} \mathrm{P}_{7 / 2}$ and ${ }^{6} \mathrm{P}_{5 / 2}$ levels of $\Delta E_{21}=(502 \pm 11)$ $\mathrm{cm}^{-1}$, again in excellent agreement with the value of $506 \mathrm{~cm}^{-1}$ that was spectroscopically deduced from the emission spectra. With this energy gap, the most suitable temperature range for high-precision luminescence thermometry is between 213 and $364 \mathrm{~K}$ with corresponding relative sensitivities of $S_{\mathrm{r}}(213 \mathrm{~K})=1.60 \% \mathrm{~K}^{-1}$ and $S_{\mathrm{r}}(364 \mathrm{~K})=0.55 \% \mathrm{~K}^{-1}$. These relative sensitivities are almost one order of magnitude higher than the corresponding sensitivities for thermometry with crystal field splitting of $72 \mathrm{~cm}^{-1}$ in this temperature range. This result demonstrates that the concept of multiple excited states with different energy separations could realize 
a

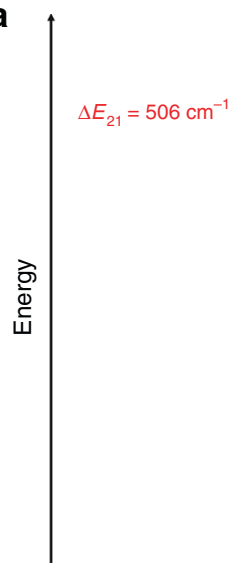

C

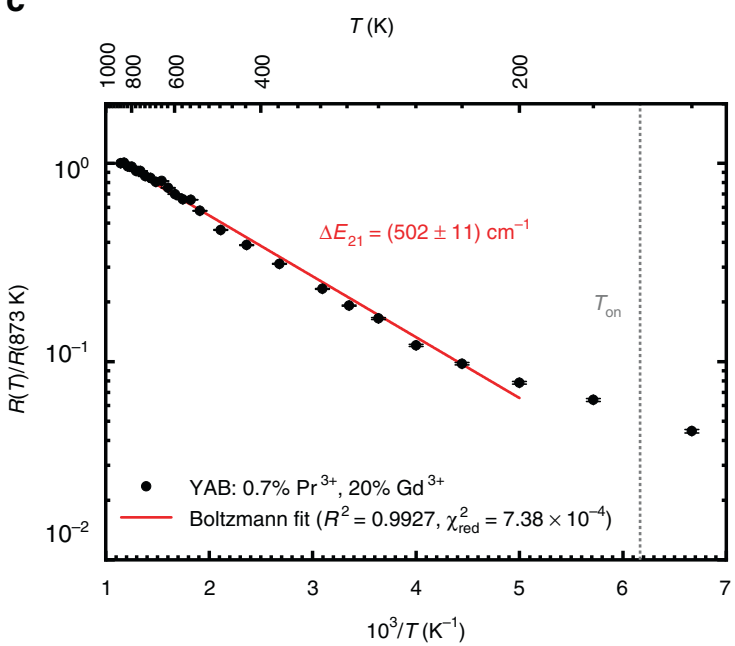

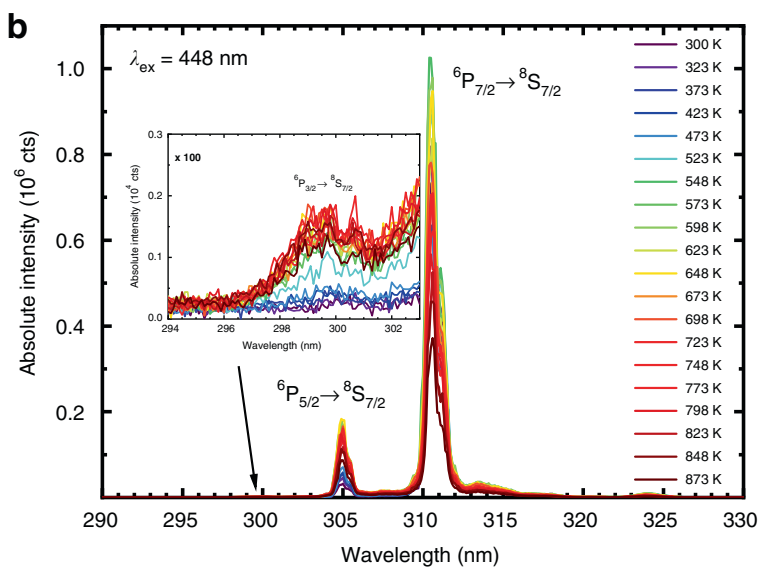

d

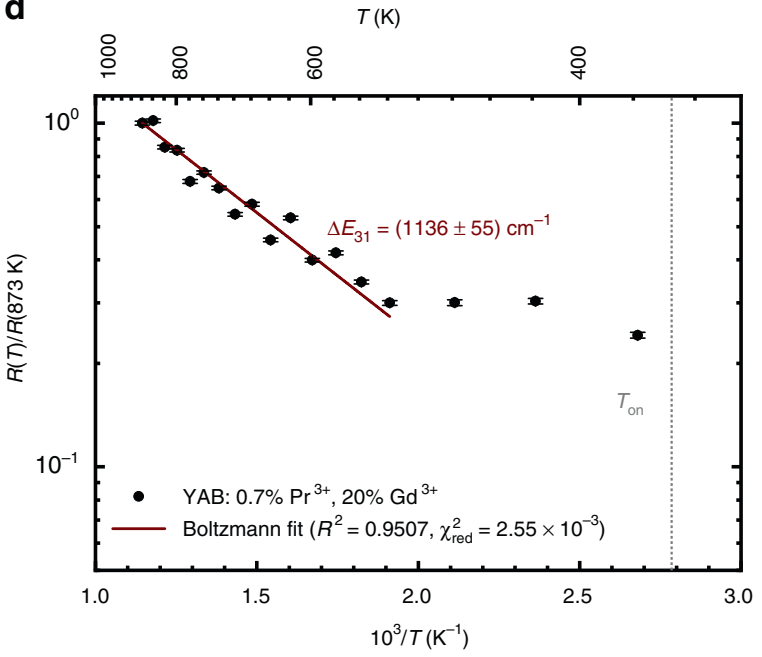

Fig. 3 Boltzmann thermometry was thermodynamically optimized at room temperature and higher temperatures $(T>500 \mathrm{~K})$ with $\mathrm{Gd}{ }^{3+}$ in $\mathrm{YAB}: \mathrm{Pr}^{3+}, \mathrm{Gd}^{3+}$. a Spin-orbit level scheme and respective radiative transitions depicting the concept for high-precision Boltzmann thermometry with $\mathrm{Gd}^{3+}$. b High-resolution upconversion photoluminescence spectra of $\mathrm{YAB}: 0.7 \% \mathrm{Pr}^{3+}, 20 \% \mathrm{Gd}^{3+}$ upon excitation of $\mathrm{Pr}^{3+}$ at $448 \mathrm{~nm}$ showing the radiative transitions from the different ${ }^{6} \mathrm{P}$, levels $(J=7 / 2,5 / 2$, and $3 / 2)$ of $\mathrm{Gd}^{3+}$. The spectra were normalized with respect to the highest intensity peak ( $I_{10} \sim 10^{6}$ counts) to demonstrate the thermally induced intensity increase of the higher energetic emission peaks. c Boltzmann plot of the temperature-dependent LIR employing the transitions from the ${ }^{6} \mathrm{P}_{5 / 2}$ and ${ }^{6} \mathrm{P}_{7 / 2}$ levels, respectively, normalized to its value at $873 \mathrm{~K}$. The fitted energy gap, statistical figures of merit and expected onset temperature for Boltzmann behaviour (Eq. (5)) are indicated. $\mathbf{d}$ Boltzmann plot of the temperaturedependent $L I R$, which employs the transitions from the ${ }^{6} \mathrm{P}_{3 / 2}$ and ${ }^{6} \mathrm{P}_{7 / 2}$ levels, normalized to its value at $873 \mathrm{~K}$. The fitted energy gap, statistical figures of merit, and expected onset temperature for Boltzmann behaviour (Eq. (5)) are indicated

high-temperature sensing accuracy over a wider temperature range, given sufficiently high photon counts of the emission bands of interest. As in the case of cryothermometry, the LIR deviates from Boltzmann behaviour below $200 \mathrm{~K}$, which is a consequence of the very slow nonradiative absorption in that temperature range $\mathrm{e}^{53-55}$. This observation also coincides very well with the estimated onset temperature for Boltzmann behaviour at $T_{\mathrm{on}}=163 \mathrm{~K}$ according to Eq. (5) for an energy gap of $506 \mathrm{~cm}^{-1}$.

An extension of the concept of optimized luminescence thermometry with $\mathrm{Gd}^{3+}$ to even higher temperatures is finally possible by the exploitation of thermal coupling between the excited ${ }^{6} \mathrm{P}_{3 / 2}$ and ${ }^{6} \mathrm{P}_{7 / 2}$ levels (Fig. $3 \mathrm{~d}$ ). The corresponding LIR is clearly only a useful temperature measure at very high temperatures due to the generally low intensity of the ${ }^{6} \mathrm{P}_{3 / 2} \rightarrow{ }^{8} \mathrm{~S}_{7 / 2}$-based transition located at $295 \mathrm{~nm}$ (inset of Fig. 3b). In particular, this application also demonstrates why $\mathrm{Gd}^{3+}$ is a particularly useful choice as a luminescent thermometer for high-temperature sensing. Since there are no lower-lying energy levels between the ${ }^{6} \mathrm{P}_{7 / 2}$ level located approximately $32000 \mathrm{~cm}^{-1}$ above the ${ }^{8} \mathrm{~S}_{7 / 2}$ ground state, thermal quenching of the $\mathrm{Gd}^{3+}$-based luminescence in a wide bandgap host compound such as YAB cannot take place. Fitting the temperature dependence of the corresponding LIR that stems from the two excited levels ${ }^{6} \mathrm{P}_{3 / 2}$ and ${ }^{6} \mathrm{P}_{7 / 2}$ to Eq. (2) 

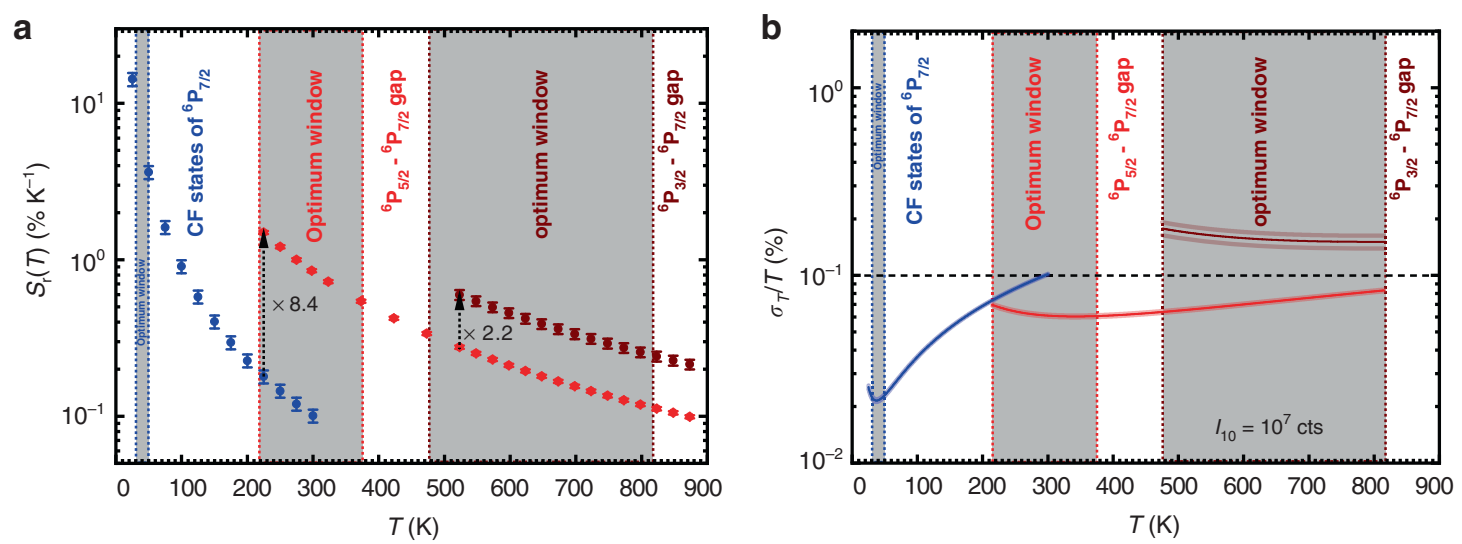

Fig. 4 Assessment of the optimum performance of a wide-range multilevel luminescent Boltzmann thermometer based on $\mathrm{Gd}^{3+}$ in $\mathrm{YAB}$ : $\mathrm{Pr}^{3+}, \mathbf{G d}^{3+}$. a Plot of the relative sensitivities $S_{r}$ of the different temperature measures and their improvement upon thermodynamically guided change of the respective energy gap of the excited levels of $\mathrm{Gd}^{3+}$ used for temperature sensing. $\mathbf{b}$ Overall theoretical relative temperature uncertainty of the different energy gaps assuming a constant integrated intensity measure of $I_{10}=10^{7}$ counts of the selected lowest energetic emission. A relative temperature uncertainty of $0.1 \%$ is desirable because it allows the measurement of temperatures below $1000 \mathrm{~K}$ with a statistical error of $\pm 1 \mathrm{~K}$. The shaded areas indicate the statistical fluctuations in the relative temperature uncertainty

(Fig. 3d) gives an effective energy gap of $\Delta E_{31}=(1136 \pm$ 81) $\mathrm{cm}^{-1}$, again in excellent agreement with the spectroscopically determined value of $1120 \mathrm{~cm}^{-1}$. This energy gap is optimally suited to measure temperatures between 472 and $805 \mathrm{~K}$ with corresponding relative sensitivities of $S_{\mathrm{r}}(472 \mathrm{~K})=0.73 \% \mathrm{~K}^{-1}$ and $S_{\mathrm{r}}(805 \mathrm{~K})=0.25 \% \mathrm{~K}^{-1}$, which is an improvement by a factor of 2.2 compared to thermometry with the ${ }^{6} \mathrm{P}_{5 / 2}-{ }^{6} \mathrm{P}_{7 / 2}$ gap in this temperature range. Here, again, thermalization and the sustainment of a Boltzmann equilibrium between the ${ }^{6} \mathrm{P}_{3 / 2}$ and ${ }^{6} \mathrm{P}_{7 / 2}$ spin-orbit levels of $\mathrm{Gd}^{3+}$ is only observed above temperatures of at least $450 \mathrm{~K}$, while Eq. (5) suggests an expected onset temperature of approximately $360 \mathrm{~K}$. The discrepancy between observed and estimated onset is related to the weak intensity of the ${ }^{6} \mathrm{P}_{3 / 2} \rightarrow{ }^{8} \mathrm{~S}_{7 / 2}$ transition that makes it difficult to accurately determine the LIR below $450 \mathrm{~K}$.

The photoluminescence spectra reveal that the intensity of the radiative emission from the ${ }^{6} \mathrm{P}_{3 / 2}$ spin-orbit level of $\mathrm{Gd}^{3+}$ is very weak compared to the emission from the ${ }^{6} \mathrm{P}_{7 / 2}$ level. According to calculations by Detrio on $\mathrm{Gd}^{3+}$, the ${ }^{6} \mathrm{P}_{3 / 2} \rightarrow{ }^{8} \mathrm{~S}_{7 / 2}$ transition is generally characterized by very low intensities irrespective of the chosen host material given the very low reduced matrix elements governing the intensities of dipole-allowed electronic transitions in Judd-Ofelt theory ${ }^{82}$. This trend raises the question of whether the LIR between the radiative transitions from the thermally coupled ${ }^{6} \mathrm{P}_{3 / 2}$ and ${ }^{6} \mathrm{P}_{7 / 2}$ levels actually offers a more precise temperature measure based on the higher relative sensitivity $S_{\mathrm{r}}(T)$ or whether continuous exploitation of the LIR between the transitions from the ${ }^{6} \mathrm{P}_{5 / 2}$ and ${ }^{6} \mathrm{P}_{7 / 2}$ levels up to higher temperatures is more favourable based on the higher luminescence intensity of the ${ }^{6} \mathrm{P}_{5 / 2} \rightarrow{ }^{8} \mathrm{~S}_{7 / 2}$ radiative transition. For that purpose, we calculated the minimum theoretical relative temperature uncertainty for both thermometry measures within the optimum temperature range of the ${ }^{6} \mathrm{P}_{3 / 2}-{ }^{6} \mathrm{P}_{7 / 2}$ gap $(472-805 \mathrm{~K})$. The relative temperature precision of a ratiometric Boltzmann-based thermometer is given by ${ }^{55}$

$$
\frac{\sigma_{T}}{T}=\frac{k_{\mathrm{B}} T}{\Delta E_{m 1}} \frac{1}{\sqrt{I_{10}}} \sqrt{1+\frac{1}{R(T)}}=\frac{k_{\mathrm{B}} T}{\Delta E_{m 1}} \frac{1}{\sqrt{I_{10}}} \sqrt{1+\frac{1}{R(\infty)} \exp \left(\frac{\Delta E_{m 1}}{k_{\mathrm{B}} T}\right)}
$$

where $I_{10}$ denotes the intensity (in integrated photon counts) of the lower energetic emission and $R(\infty)$ is the extrapolated LIR at infinite temperatures that can be obtained from the respective Boltzmann fits. Graphs depicting the evolution of both the relative sensitivity and the relative temperature uncertainty at different temperatures are shown in Fig. 4a, b. They clearly indicate that upon setting the same photon count number of $I_{10}=$ $10^{7}$ counts of the ${ }^{6} \mathrm{P}_{7 / 2} \rightarrow{ }^{8} \mathrm{~S}_{7 / 2}$-based emission, the LIR between the radiative transitions from ${ }^{6} \mathrm{P}_{5 / 2}$ and ${ }^{6} \mathrm{P}_{7 / 2}$ still gives rise to a lower relative temperature uncertainty (ca. $0.08 \%$ ) based on the higher relative intensity of the ${ }^{6} \mathrm{P}_{5 / 2} \rightarrow{ }^{8} \mathrm{~S}_{7 / 2}$ transition than the corresponding LIR involving the radiative transition from the higher energetic ${ }^{6} \mathrm{P}_{3 / 2}$ level (relative temperature uncertainty of ca. $0.2 \%$ ). In that case, it is advisable to still retain the LIR stemming from the adjacent ${ }^{6} \mathrm{P}_{5 / 2}$ and ${ }^{6} \mathrm{P}_{7 / 2}$ levels. Thus, it depends on the trade-off between practically achievable intensities and thermodynamically guided optimum temperature range, which is the more advisable choice as a temperature measure to probe high temperatures with $\mathrm{Gd}^{3+}$ in YAB: 
$\mathrm{Pr}^{3+}, \mathrm{Gd}^{3+}$. Irrespective of the choice, it is possible to keep the absolute temperature uncertainty below a threshold of $1.3 \mathrm{~K}$ even at $820 \mathrm{~K}$. We do stress, however, that these relative temperature uncertainties are purely statistical and do not include any additional systematic error that could occur in a real-case application. It is also important that these low uncertainties require a high integrated intensity $\left(\sim 10^{7}\right.$ cts $)$ of the ${ }^{6} \mathrm{P}_{7 / 2} \rightarrow{ }^{8} \mathrm{~S}_{7 / 2}$-related emission of $\mathrm{Gd}^{3+}$, which may be difficult to achieve in an application based on the proposed upconversion.

\section{Discussion}

Single ion luminescence thermometry based on a Boltzmann equilibrium between two emitting excited levels is both thermodynamically and kinetically limited to work with optimized precision only within a certain temperature range. If a luminescent thermometer is supposed to cover wider temperature ranges, thermal coupling between more than two excited states must be exploited. In this work, we offer quantitative guidelines based on a recently developed theoretical framework that allows us to extend the usability of a Boltzmann-based luminescent thermometer over wider temperature ranges by carefully choosing the energy gaps between multiple thermally coupled levels. We demonstrate the concept practically for the case of $\mathrm{Gd}^{3+}$, which exhibits three energetically well-isolated ${ }^{6} \mathrm{P}_{J}(J=7 / 2,5 / 2,3 / 2)$ levels in the UV range that can additionally rely on splitting in a local crystal field. $\mathrm{Gd}^{3+}$ is thus a prime choice for especially high-temperature ratiometric luminescence thermometry, as the black-body background does not interfere in this emission range. Moreover, it absorbs and emits in the solar-blind spectral range, and thus, any background signal from ambient sunlight is also avoided. Since $\mathrm{Gd}^{3+}$ is a weak absorber, we demonstrated an alternative excitation scheme with a cost-effective and well-established excitation wavelength of $450 \mathrm{~nm}$ available from blue LEDs via $\mathrm{Pr}^{3+}$ in a blue-to-UV upconversion process. By ground state absorption into the ${ }^{3} \mathrm{P}_{2}$ level, followed by efficient excited-state absorption into the $4 \mathrm{f}^{1} 5 \mathrm{~d}^{1}$ configuration of $\mathrm{Pr}^{3+}$, it is possible to indirectly excite $\mathrm{Gd}^{3+}$ by energy transfer into its ${ }^{6} \mathrm{I}_{J^{\prime}}\left(J^{\prime}=17 / 2 \ldots 7 / 2\right)$ levels, from which the excited state population finally undergoes nonradiative relaxation into the ${ }^{6} \mathrm{P}_{J}$ levels. Optimized Boltzmann cryothermometry between 30 and $50 \mathrm{~K}$ was possible with two crystal field components of the excited ${ }^{6} \mathrm{P}_{7 / 2}$ level separated by $72 \mathrm{~cm}^{-1}$. Temperature sensing in the range of room temperature is better performed by exploiting the LIR of the radiative transitions stemming from the ${ }^{6} \mathrm{P}_{5 / 2}$ and ${ }^{6} \mathrm{P}_{7 / 2}$ spin-orbit levels of $\mathrm{Gd}^{3+}$ separated by $506 \mathrm{~cm}^{-1}$. Finally, high-temperature thermometry between 470 and $800 \mathrm{~K}$ is also possible using Boltzmann thermalization between the ${ }^{6} \mathrm{P}_{3 / 2}$ and ${ }^{6} \mathrm{P}_{7 / 2}$ levels of $\mathrm{Gd}^{3+}$, with an energy separation of
$1120 \mathrm{~cm}^{-1}$. Both the ${ }^{6} \mathrm{P}_{3 / 2} \rightarrow{ }^{8} \mathrm{~S}_{7 / 2^{-}}$and the ${ }^{6} \mathrm{P}_{5 / 2} \rightarrow{ }^{8} \mathrm{~S}_{7 / 2^{-}}$ based emissions were compared in terms of the overall achievable temperature precision of the respective ratiometric thermometry approaches. While the ${ }^{6} \mathrm{P}_{3 / 2} \rightarrow{ }^{8} \mathrm{~S}_{7 / 2^{-}}$ based emission is very low in intensity, it offers the necessary high relative sensitivity at high temperatures. It was shown that the final choice for high-temperature thermometry with $\mathrm{Gd}^{3+}$ depends on the practically achievable integrated intensity of the lowest energetic ${ }^{6} \mathrm{P}_{7 / 2} \rightarrow{ }^{8} \mathrm{~S}_{7 / 2}$ transition.

In summary, a theoretical framework is presented to quantitatively understand and design single ion thermometers relying on multiple thermally coupled excited states to widen the window for accurate thermal sensing. The concept is shown to be feasible using a $\mathrm{Pr}^{3+}, \mathrm{Gd}^{3+}$ coactivated $\mathrm{YAl}_{3}\left(\mathrm{BO}_{3}\right)_{4}$ phosphor with $\mathrm{Gd}^{3+}$ as a Boltzmann-based single ion luminescent thermometer. UV upconversion emission from the ${ }^{6} \mathrm{P}_{J}$ levels of $\mathrm{Gd}^{3+}$ is sensitized via two-step excitation of $\mathrm{Pr}^{3+}$ at $450 \mathrm{~nm}$ to the states of the $4 \mathrm{f}^{1} 5 \mathrm{~d}^{1}$ configuration, followed by energy transfer to $\mathrm{Gd}^{3+}$, which is practically feasible with the use of high-power blue LEDs. Analysis of the temperaturedependent emission demonstrates how simple thermodynamic principles can be applied to circumvent the fundamental limitations in temperature precision of a conventional two-level single ion luminescent thermometer to finally make one thermometry system applicable to a wide range of temperatures while retaining high precision. This work paves the way for the targeted design of luminescent thermometers tailored towards applicational requirements for temperature accuracy within specific temperature ranges.

\section{Methods}

\section{Chemical reagents}

$\mathrm{Y}\left(\mathrm{NO}_{3}\right)_{3} \cdot 6 \mathrm{H}_{2} \mathrm{O}$ (Alfa Aesar, Germany, $99.99 \%$ ), $\mathrm{Al}\left(\mathrm{NO}_{3}\right)_{3}$. $9 \mathrm{H}_{2} \mathrm{O}$ (Sigma-Aldrich, Germany, $\geq 98 \%$ ), $\operatorname{Pr}\left(\mathrm{NO}_{3}\right)_{3} \cdot 6 \mathrm{H}_{2} \mathrm{O}$ (Strem Chemicals, France, 99.9\%), $\mathrm{Gd}\left(\mathrm{NO}_{3}\right)_{3} \cdot 6 \quad \mathrm{H}_{2} \mathrm{O}$ (Sigma-Aldrich, Germany, 99.99\%), $\mathrm{H}_{3} \mathrm{BO}_{3}$ (Merck, Germany, 99.8\%) and urea (Sigma-Aldrich, Germany, $\geq 98 \%$ ) were used without further purification.

\section{Synthesis of $\mathrm{YAl}_{3}\left(\mathrm{BO}_{3}\right)_{4}(\mathrm{YAB}): x \% \mathrm{Pr}^{3+}, y \% \mathrm{Gd}^{3+}$ microcrystalline powder}

Microcrystalline powder samples of YAB: $x \% \operatorname{Pr}^{3+}, y \%$ $\mathrm{Gd}^{3+}(x=0.1,0.3,0.5,0.7,1.0$, and 2.0; $y=5,10,20$, 40 , and 60) were prepared by a modified urea-nitrate solution-based combustion route ${ }^{83}$. Stoichiometric amounts of $\mathrm{Ln}\left(\mathrm{NO}_{3}\right)_{3} \cdot 6 \mathrm{H}_{2} \mathrm{O}(\mathrm{Ln}=\mathrm{Y}, \mathrm{Pr}$, and $\mathrm{Gd})$ and $\mathrm{Al}\left(\mathrm{NO}_{3}\right)_{3} \cdot 9 \quad \mathrm{H}_{2} \mathrm{O}$ were dissolved in distilled $\mathrm{H}_{2} \mathrm{O}$ (approximately $40 \mathrm{ml}$ ) under vigorous stirring at room temperature. Subsequently, solid urea (molar ratio urea/lanthanide ions $=3: 1)$ and $\mathrm{H}_{3} \mathrm{BO}_{3}(5 \mathrm{~mol} \%$ excess) were added to the transparent nitrate-containing 
solution. The solution was heated to $80^{\circ} \mathrm{C}$ to improve the solubility of the $\mathrm{H}_{3} \mathrm{BO}_{3}$ and constantly stirred for $30 \mathrm{~min}$ at that temperature. The beaker was covered with parafilm to prevent excessive solvent evaporation. The resulting solution was quickly transferred to an alumina crucible and placed into a preheated furnace at $500{ }^{\circ} \mathrm{C}$ in air for $10 \mathrm{~min}$ to initiate combustion. The thus-formed colourless solid precursor was carefully ground with additional solid $\mathrm{H}_{3} \mathrm{BO}_{3}$ (half of the previous stoichiometric amount) in a mortar to account for the losses that occurred during the combustion step. The solid mixture was finally sintered at $1100^{\circ} \mathrm{C}$ for $6 \mathrm{~h}$ in air. After naturally cooling to room temperature, the obtained colourless residue was ground to a fine powder, and its phase purity was verified by powder X-ray diffraction (Philips PW391, $\mathrm{Cu} K_{\alpha 1}$ radiation $(\lambda=$ $1.54056 \AA$ ) $, U=40 \mathrm{kV}, I=20 \mathrm{~mA}$, reflection mode). The $\mathrm{X}$-ray diffraction pattern was scanned in a $2 \theta$ range between $10^{\circ}$ and $80^{\circ}$ with a step size of $0.02^{\circ}$ (see Supplementary Information, Fig. S5). Morphology and energy dispersive $\mathrm{X}$-ray spectroscopy characterizations were performed using scanning electron microscopy (SEM; Nova, NANO SEM 430; see Supplementary Information, Fig. S6).

\section{Optical spectroscopy and temperature-dependent measurements}

Excitation was performed with an external pulsed wavelength tuneable Opotek Opolette HE 355 II (Carlsbad, CA, USA) OPO pumped by a frequency-tripled Nd: YAG laser at a repetition rate of $20 \mathrm{~Hz}$ and a temporal pulse width of approximately $6 \mathrm{~ns}$. Emission spectra were acquired on an Edinburgh FLS920 spectrofluorometer (Livingston, UK) equipped with a $0.25 \mathrm{~m}$ double Littrowconfiguration grating monochromator blazed at $300 \mathrm{~nm}$ and a Hamamatsu R928 (Shizuoka, Japan) photomultiplier tube (PMT) for photon detection. All emission spectra were corrected for grating efficiency and detector sensitivity. Temperature-dependent measurements below room temperature were performed with an Oxford Instruments liquid He flow cryostat (Oxford, UK) and an external temperature control unit, which measured the temperature by means of a thermocouple in direct contact with the powder sample holder. High-temperature luminescence spectra were acquired by placing the sample into an externally water-cooled Linkam (Surrey, UK) THMS600 microscope stage $\left( \pm 0.1^{\circ} \mathrm{C}\right.$ temperature stability). The temperature was externally controlled by a thermocouple in immediate contact with the sample holder. Photoluminescence decay curves were acquired by pulsed excitation with the OPO and detection of the timeresolved signal with a multichannel scaler (MCS) attached to a Hamamatsu H7422 PMT (Shizuoka, Japan) for minimized background. For the demonstration of the excitation of the upconverted emission of $\mathrm{Gd}^{3+}$ with blue light, a CW laser of $450 \mathrm{~nm}$ and maximum output power of $1 \mathrm{~W}$ (Changchun New Industries Optoelectronics Technology, Changchun, China) was employed as an excitation source.

\section{Acknowledgements}

D.Y. is grateful to Prof. Wei Wei from Nanjing University of Posts and Telecommunication for useful suggestions. Moreover, Q.Z. acknowledges funding from the National Science Foundation of China (U1601205, 51472088, and 51125005), while D.Y. and A.M. acknowledge funding from the Nederlandse Organisatie voor Wetenschappelijk Onderzoek (NWO, Grant No. 731.017.302). A.M. and M.S. additionally gratefully acknowledge funding from the EU-Horizon 2020 FET-Open project NanoTBTech (grant agreement no.: 801305).

\section{Author details}

${ }^{1}$ Engineering Research Center of Optical Instrument and System, The Ministry of Education, Shanghai Key Laboratory of Modern Optical Systems, University of Shanghai for Science and Technology, Shanghai 200093, China. ${ }^{2}$ Condensed Matter and Interfaces, Debye Institute for Nanomaterials Science, Department of Chemistry, Utrecht University, Princetonplein 1, 3584 CC Utrecht, The Netherlands. ${ }^{3}$ School of Materials Science and Engineering, Liaocheng University, Liaocheng 252059, China. ${ }^{4}$ State Key Laboratory of Luminescent Materials and Devices, and Institute of Optical Communication Materials, South China University of Technology, Guangzhou 510641, China. ${ }^{5}$ Inorganic Photoactive Materials, Institute of Inorganic Chemistry, Heinrich Heine University Düsseldorf, Universitätsstraße 1, 40225 Düsseldorf, Germany

\section{Author contributions}

A.M. and M.S. conceived the thermometry approach, while D.Y., A.M., and M.S. gave the input for the indirect upconversion excitation scheme. D.Y., H.L., and M.S. performed the synthesis, characterization, and optical experiments. D.Z. and Q.Z. contributed to the interpretation of the analysis results. M.S. provided the theoretical model and thermodynamic guidelines and performed the thermometric data analysis. D.Y., A.M., and M.S. wrote the manuscript. All authors contributed to the interpretation and discussion of the results.

Funding

Open Access funding enabled and organized by Projekt DEAL.

Conflict of interest

The authors declare no conflict of interest.

Supplementary information The online version contains supplementary material available at https://doi.org/10.1038/s41377-021-00677-5.

Received: 2 August 2021 Revised: 28 October 2021 Accepted: 4 November 2021

Published online: 22 November 2021

\footnotetext{
References

1. Lahiri, B. B. et al. Medical applications of infrared thermography: a review. Infrared Phys. Technol. 55, 221-235 (2012).

2. Bagavathiappan, S. et al. Infrared thermography for condition monitoring-a review. Infrared Phys. Technol. 60, 35-55 (2013).

3. Xiao, Y. Z. et al. Precision measurements of temperature-dependent and nonequilibrium thermal emitters. Laser Photonics Rev. 14, 1900443 (2020).

4. Brites, C. D. S. et al. Thermometry at the nanoscale. Nanoscale 4, 4799-4829 (2012).

5. Jaque, D. \& Vetrone, F. Luminescence nanothermometry. Nanoscale 4, 4301-4326 (2012)

6. Dramićanin, M. Luminescence Thermometry: Methods, Materials, and Applications (Woodhead Publishing, 2018).

7. Allison, S. W. \& Gillies, G. T. Remote thermometry with thermographic phosphors: instrumentation and applications. Rev. Sci. Instrum. 68, 2615-2650 (1997).
} 
8. Allison, S. W. et al. Nanoscale thermometry via the fluorescence of YAG:Ce phosphor particles: measurements from 7 to $77^{\circ} \mathrm{C}$. Nanotechnology $\mathbf{1 4}$ 859-863 (2003).

9. Allison, S. W. A brief history of phosphor thermometry. Meas. Sci. Technol. $\mathbf{3 0}$ 072001 (2019).

10. Kucsko, G. et al. Nanometre-scale thermometry in a living cell. Nature $\mathbf{5 0 0}$ 54-58 (2013).

11. Neumann, P. et al. High-precision nanoscale temperature sensing using single defects in diamond. Nano Lett. 13, 2738-2742 (2013).

12. Wang, N. et al. Magnetic criticality enhanced hybrid nanodiamond thermometer under ambient conditions. Phys. Rev. X 8, 011042 (2018).

13. Walker, G. W. et al. Quantum-dot optical temperature probes. Appl. Phys. Lett. 83, 3555-3557 (2003).

14. Ortega-Rodríguez, A. et al. 10-fold quantum yield improvement of $\mathrm{Ag}_{2} \mathrm{~S}$ nanoparticles by fine compositional tuning. ACS Appl. Mater. Interfaces 12, 12500-12509 (2020)

15. Santos, H. D. A. et al. Ultrafast photochemistry produces superbright short-wave infrared dots for low-dose in vivo imaging. Nat. Commun. 11, 2933 (2020).

16. Yakunin, S. et al. High-resolution remote thermometry and thermography using luminescent low-dimensional tin-halide perovskites. Nat. Mater. 18 846-852 (2019).

17. Morad, V. et al. Hybrid $O D$ antimony halides as air-stable luminophores for high-spatial-resolution remote thermography. Adv. Mater. 33, 2007355 (2021)

18. Gota, C. et al. Hydrophilic fluorescent nanogel thermometer for intracellular thermometry. J. Am. Chem. Soc. 131, 2766-2767 (2009).

19. Okabe, K. et al. Intracellular temperature mapping with a fluorescent polymeric thermometer and fluorescence lifetime imaging microscopy. Nat. Commun. 3, 705 (2012).

20. Sakaguchi, R., Kiyonaka, S. \& Mori, Y. Fluorescent sensors reveal subcellular thermal changes. Curr. Opin. Biotechnol. 31, 57-64 (2015).

21. Uchiyama, S. \& Gota, C. Luminescent molecular thermometers for the ratiometric sensing of intracellular temperature. Rev. Anal. Chem. 36, 20160021 (2017)

22. Brites, C. D. S., Millán, A. \& Carlos, L. D. Lanthanides in luminescent thermometry. Handb. Phys. Chem. Rare Earths 49, 339-427 (2016).

23. Brites, C. D. S., Balabhadra, S. \& Carlos, L. D. Lanthanide-based thermometers: at the cutting-edge of luminescence thermometry. Adv. Opt. Mater. 7, 1801239 (2019).

24. Souza, A. S. et al. Highly-sensitive $\mathrm{Eu}^{3+}$ ratiometric thermometers based on excited state absorption with predictable calibration. Nanoscale 8, 5327-5333 (2016).

25. Trejgis, K., Bednarkiewicz, A. \& Marciniak, L. Engineering excited state absorption-based nanothermometry for temperature sensing and imaging. Nanoscale 12, 4667-4675 (2020).

26. Trejgis, $\mathrm{K}$ et al. $\mathrm{Nd}^{3+}$ doped TZPN glasses for $\mathrm{NIR}$ operating single band ratiometric approach of contactless temperature readout. J. Lumin. 224, 117295 (2020).

27. Brites, C. D. S. et al. Instantaneous ballistic velocity of suspended Brownian nanocrystals measured by upconversion nanothermometry. Nat. Nanotechnol. 11, 851-856 (2016)

28. Bastos, A. et al. Thermal properties of lipid bilayers determined using upconversion nanothermometry. Adv. Funct. Mater. 29, 1905474 (2019).

29. Caixeta, F. J. et al. High-quantum-yield upconverting $\mathrm{Er}^{3+} / \mathrm{Mb}^{3+}$ organic-inorganic hybrid dual coatings for real-time temperature sensing and photothermal conversion. J. Phys. Chem. C 124, 19892-19903 (2020).

30. del Rosal, B. et al. $\mathrm{Nd}^{3+}$ ions in nanomedicine: perspectives and applications. Optical Mater. 63, 185-196 (2017).

31. del Rosal, B. et al. In vivo luminescence nanothermometry: from materials to applications. Adv. Opt. Mater. 5, 1600508 (2017).

32. Piñol, R. et al. Real-time intracellular temperature imaging using lanthanidebearing polymeric micelles. Nano Lett. 20, 6466-6472 (2020).

33. Ximendes, E. C. et al. Thulium doped $\mathrm{LaF}_{3}$ for nanothermometry operating over 1000 nm. Nanoscale 11, 8864-8869 (2019).

34. Skripka, A. et al. Advancing neodymium single-band nanothermometry. Nanoscale 11, 11322-11330 (2019).

35. Hazra, C. et al. Erbium single-band nanothermometry in the third biological imaging window: potential and limitations. Adv. Opt. Mater. 8, 2001178 (2020).

36. Geitenbeek, R. G. et al. In situ luminescence thermometry to locally measure temperature gradients during catalytic reactions. ACS Catal. 8, 2397-2401 (2018).

37. van Ravenhorst, I. K. et al. In situ local temperature mapping in microscopy nano-reactors with luminescence thermometry. ChemCatChem $\mathbf{1 1}$ 5505-5512 (2019).
38. Hartman, T. et al. Operando monitoring of temperature and active species at the single catalyst particle level. Nat. Catal. 2, 986-996 (2019).

39. Geitenbeek, R. G. et al. Luminescence thermometry for in situ temperature measurements in microfluidic devices. Lab Chip 19, 1236-1246 (2109).

40. Hartman, T. et al. Operando nanoscale sensors in catalysis: all eyes on catalyst particles. ACS Nano 14, 3725-3735 (2020).

41. Bednarkiewicz, A. et al. Standardizing luminescence nanothermometry for biomedical applications. Nanoscale 12, 14405-14421 (2020).

42. Dramićanin, M. D. Trends in luminescence thermometry. J. Appl. Phys. 128, 040902 (2020).

43. Bednarkiewicz, A. et al. Luminescence based temperature bio-imaging: status, challenges, and perspectives. Appl. Phys. Rev. 8, 011317 (2021).

44. Ximendes, E. et al. Quo vadis, nanoparticle-enabled in vivo fluorescence imaging? ACS Nano 15, 1917-1941 (2021).

45. van Swieten, T. P. et al. Mapping elevated temperatures with a micrometer resolution using the luminescence of chemically stable upconversion nanoparticles. ACS Appl. Nano Mater. 4, 4208-4215 (2021).

46. Labrador-Páez, L. et al. Reliability of rare-earth-doped infrared luminescent nanothermometers. Nanoscale 10, 22319-22328 (2018).

47. Shen, Y. L. et al. In vivo spectral distortions of infrared luminescent nanothermometers compromise their reliability. ACS Nano $\mathbf{1 4}$ 4122-4133 (2020).

48. Errulat, D. et al. A luminescent thermometer exhibiting slow relaxation of the magnetization: toward self-monitored building blocks for next-generation optomagnetic devices. ACS Cent. Sci. 5, 1187-1198 (2019).

49. Gálico, D. A. et al. Triplet-state position and crystal-field tuning in optomagnetic lanthanide complexes: two sides of the same coin. Chem. A Eur. J. 25, 14625-14637 (2019).

50. Kaczmarek, A. M. et al. Developing luminescent ratiometric thermometers based on a covalent organic framework (COF). Angew. Chem. Int. Ed. 59 1932-1940 (2020).

51. Gomez, G. E. et al. Tunable energy-transfer process in heterometallic MOF materials based on 2,6-naphthalenedicarboxylate: solid-state lighting and near-infrared luminescence thermometry. Chem. Mater. 32, 7458-7468 (2020).

52. Kaczmarek, A. M. et al. Visible and NIR upconverting $\mathrm{Er}^{3+}-\mathrm{Yb}^{3+}$ luminescent nanorattles and other hybrid PMO-inorganic structures for in vivo nanothermometry. Adv. Funct. Mater. 30, 2003101 (2020).

53. Geitenbeek, R. G., de Wijn, H. W. \& Meijerink, A. Non-Boltzmann luminescence in $\mathrm{NaYF}_{4}: \mathrm{Eu}^{3+}$ : implications for luminescence thermometry. Phys. Rev. Appl. 10, 064006 (2018).

54. Suta, M. et al. Making $\mathrm{Nd}^{3+}$ a sensitive luminescent thermometer for physiological temperatures - an account of pitfalls in Boltzmann thermometry. Nanomaterials 10, 543 (2020).

55. Suta, M. \& Meijerink, A. A theoretical framework for ratiometric single ion luminescent thermometers - thermodynamic and kinetic guidelines for optimized performance. Adv. Theory Simul. 3, 2000176 (2020).

56. Tian, X. N. et al. Temperature sensor based on ladder-level assisted thermal coupling and thermal-enhanced luminescence in $\mathrm{NaYF}_{4}: \mathrm{Nd}^{3+}$. Opt. Express 22 , 30333-30345 (2014).

57. Cirić, A. et al. Comparison of three ratiometric temperature readings from the $\mathrm{Er}^{3+}$ upconversion emission. Nanomaterials 10, 627 (2020).

58. Li, L. P. Three-energy-level-cascaded strategy for a more sensitive luminescence ratiometric thermometry. Sens. Actuators A: Phys. 304, 111864 (2020).

59. Belokoneva, E. L. et al. The crystal structure of $\mathrm{YAl}_{3}\left(\mathrm{BO}_{3}\right)_{4}$. Zh. Strukturnoi Khimii 22, 196-198 (1981).

60. $\mathrm{Hu}, \mathrm{C}$. H. et al. Visible-to-ultraviolet upconversion in $\mathrm{Pr}^{3+}: \mathrm{Y}_{2} \mathrm{SiO}_{5}$ crystals. Chem. Phys. 325, 563-566 (2006).

61. Cates, E. L. \& Kim, J. H. Upconversion under polychromatic excitation: $\mathrm{Y}_{2} \mathrm{SiO}_{5}$ : $\mathrm{Pr}^{3+}, \mathrm{Li}^{+}$converts violet, cyan, green, and yellow light into UVC. Optical Mater. 35, 2347-2351 (2013).

62. Cates, E. L., Wilkinson, A. P. \& Kim, J. H. Visible-to-UVC upconversion efficiency and mechanisms of $\mathrm{Lu}_{7} \mathrm{O}_{6} \mathrm{~F}_{9}: \mathrm{Pr}^{3+}$ and $\mathrm{Y}_{2} \mathrm{SiO}_{5}: \mathrm{Pr}^{3+}$ ceramics. J. Lumin. 160, 202-209 (2015).

63. Cates, E. L. \& Li, F. F. Balancing intermediate state decay rates for efficient $\mathrm{Pr}^{3+}$ visible-to-UVC upconversion: the case of $\beta-\mathrm{Y}_{2} \mathrm{Si}_{2} \mathrm{O}_{7}: \mathrm{Pr}^{3+}$. RSC Adv. $\mathbf{6}$, 22791-22796 (2016)

64. Du, Y. Y. et al. Blue-pumped deep ultraviolet lasing from lanthanide-doped $\mathrm{Lu}_{6} \mathrm{O}_{5} \mathrm{~F}_{8}$ upconversion nanocrystals. Adv. Opt. Mater. 8, 1900968 (2020).

65. Sahu, S. P. et al. The myth of visible light photocatalysis using lanthanide upconversion materials. Environ. Sci. Technol. 52, 2973-2980 (2018). 
66. Harada, N. et al. Discovery of Key TIPS-naphthalene for efficient visible-to-UV photon upconversion under sunlight and room light. Angew. Chem. Int. Ed. 60 142-147 (2021).

67. Back, M. et al. Effective ratiometric luminescent thermal sensor by $\mathrm{Cr}^{3+}$-doped mullite $\mathrm{Bi}_{2} \mathrm{Al}_{4} \mathrm{O}_{9}$ with robust and reliable performances. Adv. Optical Mater. 8 , 2000124 (2020)

68. Back, $\mathrm{M}$. et al. Pushing the limit of Boltzmann distribution in $\mathrm{Cr}^{3+}$-doped $\mathrm{CaHFO}_{3}$ for cryogenic thermometry. ACS Appl. Mater. Interfaces 12, 38325-38332 (2020).

69. Mykhaylyk, V. et al. Multimodal non-contact luminescence thermometry with Cr-doped oxides. Sensors 20, 5259 (2020).

70. Sytsma, J., Imbusch, G. F. \& Blasse, G. The spectroscopy of $\mathrm{Gd}^{3+}$ in yttriumoxychloride: Judd-Ofelt parameters from emission data. J. Chem. Phys. 91 1456-1461 (1989).

71. Sytsma, J. \& Blasse, G. Comparison of the emission of $\mathrm{Eu}^{2+}$ in $\mathrm{MFCl}(\mathrm{M}=\mathrm{Sr}, \mathrm{Ba})$ and $G d^{3+}$ in YOCl. J. Lumin. 51, 283-292 (1992).

72. Bartl, M. H. et al. Growth, optical spectroscopy and crystal field investigation of $\mathrm{YAl}_{3}\left(\mathrm{BO}_{3}\right)_{4}$ single crystals doped with tripositive praseodymium. Spectrochim. Acta Part A: Mol. Biomol. Spectrosc. 57, 1981-1990 (2001).

73. de Vries, A. J. \& Blasse, G. On the possibility to sensitize $\mathrm{Gd}^{3+}$ luminescence by the $\mathrm{Pr}^{3+}$ ion. Mater. Res. Bull. 21, 683-694 (1986).
74. Balabhadra, S. et al. Upconverting nanoparticles working as primary thermometers in different media. J. Phys. Chem. C 121, 13962-13968 (2017).

75. Sytsma, J. et al. Spectroscopic studies and crystal-field analysis of $\mathrm{Cm}^{3+}$ and $\mathrm{Gd}^{3+}$ in $\mathrm{LuPO}_{4}$. Phys. Rev. B 52, 12668-12676 (1995).

76. Riseberg, L. A. \& Moos, H. W. Multiphonon orbit-lattice relaxation in $\mathrm{LaBr}_{3}$, $\mathrm{LaCl}_{3}$, and $\mathrm{LaF}_{3}$. Phys. Rev. Lett. 19, 1423-1426 (1967).

77. Riseberg, L. A. \& Moos, H. W. Multiphonon orbit-lattice relaxation of excited states of rare-earth ions in crystals. Phys. Rev. J. Arch. 174, 429-438 (1968),

78. Back, M. et al. Boltzmann thermometry in $\mathrm{Cr}^{3+}$-doped $\mathrm{Ga}_{2} \mathrm{O}_{3}$ polymorphs: the structure matters! Adv. Opt. Mater. 9, 2100033 (2021).

79. Liu, X. et al. Mixed-lanthanoid metal-organic framework for ratiometric cryogenic temperature sensing. Inorg. Chem. 54, 11323-11329 (2015).

80. Ananias, D. et al. Near-infrared ratiometric luminescent thermometer based on a new lanthanide silicate. Chem. A Eur. J. 24, 11926-11935 (2018).

81. Abram, C. et al. $\mathrm{SCVO}_{4}: \mathrm{Bi}^{3+}$ thermographic phosphor particles for fluid temperature imaging with sub- ${ }^{\circ} \mathrm{C}$ precision. Opt. Lett. 45, 3893-3896 (2020).

82. Detrio, J. A. Line strengths for $\mathrm{Gd}^{3+}$ at a $C_{4 v}$ site in $\mathrm{SrF}_{2}$. Phys. Rev. B 4, 1422-1427 (1971)

83. Yu, D. C. et al. Understanding and tuning blue-to-near-infrared photon cutting by the $\mathrm{Tm}^{3+} / \mathrm{Yb}^{3+}$ couple. Light:: Sci. Appl. 9, 107 (2020). 